\title{
Minimum Equipment List as a Mechanism of Motion in MIRCE Mechanics
}

\author{
Knezevic J* \\ Department of mechanics, MIRCE Akademy, UK
}

Submission: February 02, 2018; Published: March 29, 2018

"Corresponding author: J Knezevic, MIRCE Akademy, Woodbury Park, Exeter, EX5 1JJ, UK, Email: jk@mirceakademy.com

\begin{abstract}
To avoid the loss of consumer trust, revenue or capability any disruption to a system's functionality is unacceptable to the providers, on one hand and also to their receivers, on the other. Consequently, every effort must be made to ensure the continuity of the provision of the system's functionality through calendar time. One of the methods used to minimise disruption to operational capability, especially in the aviation industry, was the creation of the Minimum Equipment List (MEL). This list identifies the equipment/components present into system that are not necessary to be operational for the safe provision of the functionality of the system, in accordance with the prescribed operational and maintenance restrictions, and approved by the regulatory authorities. Consequently, the main objective of this paper is to present the concept of the MEL as one of the potential mechanisms to be used outside aviation community to further influences the motion of a functionable system type through MIRCE Space and potentially enhance its functionability performance as perceived by MIRCE Science.
\end{abstract}

Keywords : Electron; Positive functionability action; Positive functionability state; Negative functionability state; Mechanism

Abbreviations : PFS: Positive Functionability Action; NFS: Negative Functionability State; PFA: Positive Functionability Action; NFA: Negative Functionability Action; PFE: Positive Functionability Event; NFE: Negative Functionability Event

\section{Introduction}

"Motion does not mean travel of the ball-type electron along some orbit around the nucleus. Motion is the change in the state of the system "atom" in time." Werner Heisenberg

To facilitate the flow of functionality through calendar time provided by the functionable system types MIRCE Science is focused on the scientific understanding and description of the physical phenomena and human rules that govern their in-service behaviour. According to Knezevic [1], functionable system type is "a set of mutually related components uniquely put together to perform at least one measurable function and a set of functionability rules that govern its Functionability performance."

In MIRCE Science, at any instant of calendar time a given functionable system type could be in one of the following two states:

1. Positive Functionability State (PFS), a generic name for a state in which a functionable system type is able to deliver the expected measurable function(s),
2. Negative Functionability State (NFS), a generic name for a state in which a functionable system type is unable to deliver the expected measurable function(s), resulting from any reason whatsoever.

The motion of a functionable system type through the Functionability states, in the direction of calendar time, is generated by Functionability actions, which are classified as:

1. Positive Functionability Action (PFA), a generic name for any natural process or human activity that compels a system to move to a PFS.

2. Negative Functionability Action (NFA), a generic name for any natural process or human activity that compels a system to move to a NFS.

The motion of a functionable system type through the functionability states is manifested through the occurrences of Functionability events, which are classified as:

1. Positive Functionability Event (PFE), a generic name for any physically observable occurrence in time that signifies the transition of a functionable system type from a NFS to a 
PFS.

2. Negative Functionability Event (NFE), a generic name for any physically observable occurrence in time that signifies the transition of a functionable system type from a PFS to a NFS.

Transition of a functionable system type from PFS to NFS and loss of functionality is undesirable in private sector due to loss of revenue and to public sector due to loss of benefits. For example, in airline business loss of service is unacceptable due to negative consequences of the following types: [2].

1. Loss of income generated by transporting passengers and cargo

2. Poor customer relationships

3. Increased demand for support resources (spares, tools, equipment, etc).

4. Increased numbers of maintenance facilities, including skills and training of personnel required to deal with the consequences of cancellations.

5. Costs arising from re-routes, aircraft substitution, passenger handling (hotels, buses, meal vouchers).

Cancelled flights generate the cost to the customer too, due to disrupted plans, missed business appointments, lost time and poetical consequences to the cargo due to late shipments.

Generally speaking, all affords must be made to enable passengers and cargo to go to their destinations on time, in safe manner. Legendary Chief Mechanic on Boeing 777, Jack Hessburg, has immortalised this sentiment by saying, "All I want to do is to go to Cleveland on time and never crash" [1].

Consequently, the main objective of this paper is to present the concept of the MEL as one of the potential mechanisms to be used outside aviation community to further influences the motion of a functionable system type through MIRCE Space and potentially enhance its functionability performance as perceived by MIRCE Science [1].

\section{Minimum Equipment List in Commercial Aviation}

According to Hessburg [2], the structure and approval of the early Minimum Equipment Lists (MEL) lists were principally in the hands of the individual CAA Air Carrier Inspectors. Technical evaluation of items for inclusion into a list was based upon the individual inspector's knowledge, competence, and subjective analysis of a specific aircraft type. The result was that the MEL for operator " $\mathrm{A}$ " and that operator "B" (both using the same model aircraft) were frequently different; one airline's inspector being very conservative and disallowing all but the simplest of equipment to be inoperative, the other being far more liberal. Individual operators would claim favouritism when one discovered that their competitor had a less restrictive MEL.

This lack of objective analysis and standardisation of MEL resulted in the institutionalisation of the process by the mid1960s. The FAA adopted centralised control and publication of separate Master Minimum Equipment Lists (MMEL) for each large aircraft type.

Present regulations continue to recognise the original MEL concept. FARs 125, and 135 operators were included in the concept by the late 1970s. In 1991, single engine operations under FAR 135 were added to the concept. Lastly, FAR Part 91 operations are also now covered [3].

It is necessary to stress that nothing in this concept disallows the authority of the pilot-in-command. The pilot may, at his or her discretion, require that any item covered by the MEL be repaired before flight.

Operations with certain items of equipment inoperative are not considered an abrogation of the aircraft Type Certificate. When operating under Parts 121, 125 or 135, an approved Minimum Equipment List is recognised as an approved change to the type design. Therefore, the altered status of the aircraft under the MEL remains an acceptable certified configuration. Consequently, adoption of an MEL item does not require recertification of the type design [3].

Operating with an approved MEL and a letter of authorisation under FAR Part 91 constitutes a Supplemental Type Certificate for the aircraft. As such, an MEL approved under Part 91 is issued against a specific aircraft(s) i.e. aircraft serials number(s). The aircraft(s) will be listed on the cover sheet of the approved MEL.

Is necessary to stress a mechanic or inspector is not in violation of the FAR for releasing an aircraft as airworthy when certain items are inoperative under an approved MEL. Because an air carrier airworthiness release requires certification that the work performed is in accordance with the certificate holder's manual. Because an approved MEL is a part of the certificate holder's manual, a mechanic is relieved of responsibility for the inoperative status of MEL items. The actions taken under the requirements of an approved MEL would "clear" the discrepancy from the aircraft maintenance record and would consequently revalidate the maintenance release.

A mechanic is not responsible for any contingent maintenance required by the MEL for any previously deferred items unless additional or repetitive maintenance is required. An aircraft maintained under FAR Part 91 is returned to service under the provisions of FAR 43.5 and is unaffected by this dilemma as an approved MEL under Part 91 is considered a supplemental type certificate.

\section{Creation of the Master Minimum Equipment List}

During the initial design of an aircraft the development of Master Minimum Equipment List, MMEL, begins. For new designs, the manufacturer submits a preliminary list of items to be considered by the relevant aviation authorities. Aircraft operators, interested public (safety organisations, foreign regulatory agencies, union representatives, and the like), use 
this list to develop the master. The final MMEL is released, after approval by designated authority.

Once adopted, an MMEL is periodically revised. These revisions arise from individual operators petitioning the relevant authorities for additions, deletions, or clarification of items. These changes continue throughout the useful life of the aircraft type. There is no set schedule for the revisions; they take place on an as needed basis. Early in an aircraft model's life, meetings might be held yearly, whereas later in its life, the meetings can be several years apart.

\section{MMEL is for Aircraft not Airline}

The MMEL is a generic list for a given aircraft type. Thus, items are included in the master that may not be installed in a given airline's fleet. There is no system for tracking recording, crew notification and clearing of a deferred item contained in the list. The list also contains time limits on how long a given item may be deferred. It identifies requirements for placarding the cockpit. It requires which items must have maintenance and flight operations procedures or limits.

Each airline must prepare its own MEL using the master as the source. Operators are responsible for exercising the necessary control to insure that an acceptable level of safety is maintained. This includes a repair program embracing the parts, personnel, facilities, procedures, and schedules to insure timely clearance of deferred items.

An individual airline's MEL may be more restrictive but not less than the Master Minimum Equipment List. They may include, with appropriate conditions and limitations, items not contained in the master list such as equipment not required for a given flight operation; that which is more than required by the FAR; and equipment that, for internal administrative control reasons to the operator, is best placed within the context of his MEL

\section{Deferring Process under MEL}

The specific process for deferring at any given airline will differ. However, once it has been determined that an item is deferrable, a decision is made to defer or fix. This normally involves, at the minimum, station maintenance personnel and the pilot-in-command. However, in many instances, flight dispatch, maintenance engineering and a quality control organisation will be party to the decision. Some airlines designate in the body of their MEL, specific individuals or organisations with deferral authority for each item listed.

Station maintenance personnel have several responsibilities that include properly securing the deferred item, logging item correctly in required documents, notifying specific individuals and organisations to ensure that the necessary bookkeeping will take place, thus insuring that the item is properly tracked and scheduled for later repair within allowable time limits.

Dispatch and/or the pilot-in command shall, as appropriate observe any special limitations or modified operating procedures attendant to the deferred item and notify other operations organisations and down line stations that are affected by the deferral.

Maintenance control or other appropriate organisation charged with tracking deferred items and scheduling will take appropriate action to clear the item from the deferred log within the allowed time for deferral.

\section{An Example of Minimum Equipment List}

To illustrate the concept and a practical application of MEL the vacuum pump of Piper SeminolePA-44-180 is addressed, for no particular reason. It is a four seat light twin aircraft, which has been in-service since 1978. It is at twin engine development of PA-28 Archer aircraft with new T-tail and semi-tapered wings. Production ceased in 1981, restarted in 1988 and ceased once more in 1990.

Main technical and functionality characteristics of this aircraft are as following:

1. Power plants: Two $135 \mathrm{~kW}$ (180hp) Lycoming 0-360E1AD flat four piston engines driving two or optionally three blade constant speed Hartzell propellers.

2. Performance: Max speed $311 \mathrm{~km} / \mathrm{h}$ (168kt), max cruising speed $309 \mathrm{~km} / \mathrm{h}$ (167kt), long range cruising speed $280 \mathrm{~km} / \mathrm{h}$ (151kt). Initial rate of climb $1200 \mathrm{ft} / \mathrm{min}$. Service ceiling 17,100ft. Range with reserves $1630 \mathrm{~km}(880 \mathrm{~nm})$. Weights: Empty $1070 \mathrm{~kg}$ (2360lb), max takeoff $1723 \mathrm{~kg}$ (3800lb).

3. Dimensions: Wing span $11.77 \mathrm{~m}$ (38ft 8in), length $8.41 \mathrm{~m}(27 \mathrm{ft} 7 \mathrm{in})$, height $2.59 \mathrm{~m}$ (8ft $6 \mathrm{in})$. Wing area $17.1 \mathrm{~m} 2$ (183.8sq ft).

4. Production: Total Seminole production through to end 2004 amounted to 672, including 86 Turbo Seminoles.

The Piper PA-44 Master Minimum Equipment List (MMEL) for Part 91 operations lists all items of installed equipment that are permitted to be inoperative. The MMEL states, in part, that "it is incumbent on the operator to endeavour to determine if Operations and/or Maintenance] procedures for that equipment must be developed.

The MMEL is intended to permit operations with inoperative items of equipment for the minimum period of time necessary until repairs can be accomplished. It is important that repairs be accomplished at the earliest opportunity in order to return the aircraft to its design level of safety and reliability. Operators are responsible for exercising the necessary operational control to ensure that an acceptable level of safety is maintained" [4].

According to Section 37 "Vacuum/Pressure," sequence title "1. Vacuum Pump" states "two vacuum pumps are installed and one is required for dispatch. It further provided, under the "Remarks or Exceptions," that "One may be inoperative for day VFR flights". 
The PA-44 Scheduled Maintenance Manual includes a checklist to be utilised for inspections every 50 and/or $100 \mathrm{hr}$. Subsection B.36 states "Inspect and operationally test vacuum pumps and lines." It further requires that the vacuum pump installed on each engine is inspected every $100 \mathrm{hr}$. Note 7 further states "Replace or overhaul, as required, or at engine overhaul".

The Pilot's Operating Handbook states in Section 7.19. "The vacuum system operates the air-driven gyro instruments. The vacuum system consists of a vacuum pump on each engine, plus plumbing and regulating equipment. A shear drive protects the engine from damage. If the drive shears, the gyros will become inoperative. The vacuum gauge mounted on the right instrument panel to the right of the radios provides valuable information to the pilot about the operation of the vacuum system (A low vacuum indicator light is provided in the annunciator panel). In the event of any gauge variation from the norm, the pilot should have a mechanic check the system to prevent possible damage to the system components or eventual failure of the system. A vacuum regulator is provided in the system to protect the gyros. The valve is set so the normal vacuum reads 4.8 to $5.2 \mathrm{in}$. of mercury" [4].

Should suction drop below $4.5 \mathrm{in}$. Hg, pilots are cautioned to increase rpm to 2,700 , descend to maintain $4.5 \mathrm{in}$. and use the electric turn indicator to monitor directional indicator and attitude indicator performance.

\section{Impact of Minimum Equipment List on Functionability Performance}

"We did not buy the airplane to make a roost for pigeons on the vertical fin, fouling the logo. So equipment availability is a fundamental tenant which we forget about".

Bob Six, CEO Continental Airlines According to the philosophy of MIRCE Science the main objective of existence of any functional system is to do a work during the calendar time. [1] To differentiate the concept of work in physics and in MIRCE Science, the author has named the latter as a functionability work. Hence, functionability work is considered done when a system delivers a measurable function over an interval of time, in a similar way that classical physics considers work done when an external force displaces an object over a distance.

\section{Consequently, in MIRCE Science functionability work is classified as [1]:}

1. Positive Functionability Work (PFW): a generic name for the physically measurable performance of a functionable system type proportional to the duration of the calendar time during which the expected function(s) are performed, measured in hours [Hr].

2. Negative Functionability Work (NFW): a generic name for the physically measurable performance of a functionable system type proportional to the duration of the calendar time during which required positive functionability actions are performed, measured in hours [Hr].
Although the concept of functionability work is the main measure of functionability performance, it is necessary to take into account the physical resources related to the execution of functionability works, like material, personnel, spares, tools, equipment, facilities and energy and similar must be taken into account. As all of them have individual monetary values, in MIRCE Science, they are brought together under the single umbrella of functionability cost, and it is classified into following two types [1]:

1. Cost of Positive Work (CPW): a generic name for the physically measurable performance of a functionable system type determined by the monetary value of all the resources related to the delivery of positive functionability work, like operational personnel, consumable material, equipment, facilities, energy and similar. Generally speaking, it encompasses all the costs related to delivery of the positive functionability work by the functionable system type during a given interval of calendar time T, denoted as CPW (T). It is equal to the sum of the following cost elements: [1]:

$$
C P W_{S}(T)=C P W_{\text {set }}+C P W_{\text {fix }}(T)+C P W_{\text {var }}(T)[M U]
$$

Where: $C P W_{\text {set }}$ is the Set up Cost, $C P W_{f x x}(\mathrm{~T})$ is the Fixed Cost and): a $C P W_{\text {var }}$ is the Variable Cost of delivering positive work.

2. Cost of Negative Work (CNW): a generic name for the physically measurable performance of a functionable system type determined by the monetary value of all the resources used by a functionable system type to perform the negative functionability work, like spare parts, qualified personnel, material, equipment, facilities, energy and similar. Thus, it encompassed all the costs related to performing the negative functionability work, performed on the functionable system type during a given interval of calendar time $\mathrm{T}$, denoted as CNW (T), is equal to the following sum [1].

$$
C N W_{S}(T)=C N W_{\text {set }}+C N W_{f x x}(T)+C N W_{\text {var }}(T) \quad[M U]
$$

Where: $C P W_{s e t}$ is the Set up Cost, $C P W_{f i x}$ (T) is the Fixed Cost and): a $C P W_{\text {ara }}$ is the Variable Cost of delivering positive work.

The delivery of positive and negative function ability work through time uniquely determines the positive and negative function ability costs that correspond to the motion of a function able system type through the function ability states. As this motion is in the direction of calendar time, it means that the magnitudes of the positive and negative function ability costs are non-decreasing measurable characteristics of the function able system types, and as such they are "legitimate" measures of their overall function ability performance [1].

\section{Cost of Lost Revenue}

The main business of any business is staying in business. For that to happen it is essential to generate a profit, which is commonly accepted as the difference between the revenue generated and the costs incurred to generate the revenue. Generally speaking, the revenue generated by each functionable 
system type during a given interval of calendar time, denoted as REV (T), could be calculated as the product of the hourly income, denoted as $\mathrm{HI}$, expressed in $[\mathrm{MU} / \mathrm{Hr}]$ and the amount of the positive functionability work done by the system during the stated interval of calendar time, PFW (T) expressed in [Hr], thus [1]:

$$
R E V(T)=H I \times P F W_{S}(T)[M U]
$$

Financially minded analysts totally ignore the non-generated cost while the functionable system type is in the NFS during a given interval of calendar time. However, MIRCE Science this cost category has been analysed and named the Cost of Lost Revenue, CLR (T) [1]. This cost category could be even higher than that of revenue generated due to the potential consequences of the occurrences of NFEs to the business and environment.

In MIRCE Science a profit, PRF (T), is equal to the difference between the revenue, REV (T) and the Total Function ability Cost, during the stated period of calendar time, TFC(T), thus [1]:

$$
\begin{aligned}
P R F(t) & =R E V_{S}(T)-T F C_{S}(T) \\
& =\left[H I_{S} \times P F W_{S}(T)\right]-\left[C P W_{S}(T)+C N W_{S}(T)+C L R(T)\right] \quad[M U]
\end{aligned}
$$

The MIRCE Profitability Equation is the only one, known to the author, which unifies all aspects of the function ability performance of a functionable system type including the cost of lost revenue, which, in reality, is an in-separable element of the expected profit. This equation enables more accurate predictions of the expected profit to be made for each operational scenario, maintenance policy and support strategy, including the "length of MEL". Also, this equation "integrates" the decision makers of functional systems types and the decision makers of corresponding functionable system types into a single "normalised" analytical entity, rather than, as currently considered, two competing parties searching self set targets in isolation.

\section{Conclusion}

To avoid the loss of consumer trust, revenue or capability any disruption to a system's functionality is unacceptable to the providers, on one hand and also to their receivers, on the other. Consequently, every effort must be made to ensure the continuity of the provision of the system's functionality through calendar time. One of the methods used to minimise disruption to operational capability, especially in the aviation industry, was the creation of the Minimum Equipment List (MEL).

A minimum equipment list (MEL) is a list which provides for the operation of aircraft, subject to specified conditions, with particular equipment inoperative (which is) prepared by an operator in conformity with, or more restrictive than, the Master Minimum Equipment List, established for the aircraft type. (ICAO Annex 6: Operation of Aircraft)

The MMEL is a list established for a particular function able system type by the organization responsible for the type design with the approval of the State of Design which identifies items which individually may be unserviceable at the commencement of a flight. The MMEL may be associated with special operating conditions and rules.

The impact of introducing the MEL for any aircraft type could be quantified through the functionability performance measures, namely function ability work and function ability cost, positive and negative, which are fully defined by the function ability equations of MIRCE Science.

In summary, this paper has shown that the MEL as one of the potential mechanisms to influence the motion of a function able system type through MIRCE Space and as such it can enhance its functionability performance as understood through the application of MIRCE Science [1].

\section{References}

1. Knezevic J (2017) The Origin of MIRCE Science, MIRCE Science, Exeter, UK.

2. Hessburg J (2000) Minimum Equipment List, Aviation Maintenance Technology.

3. Hessburg JG (2001) Air carrier MRO Handbook, McGraw Hill, New York, USA.

4. Aarons RN (2016) In-flight Breakup, Gambling on a single vacuum pump in IFR a losing bet, Business \& Commercial Aviation.

Your next submission with Juniper Publishers
will reach you the below assets
- Quality Editorial service
- Swift Peer Review
- Reprints availability
- E-prints Service
- Manuscript Podcast for convenient understanding
- Global attainment for your research
- Manuscript accessibility in different formats
( Pdf, E-pub, Full Text, Audio)
- Unceasing customer service
Track the below URL for one-step submission
https://juniperpublishers.com/online-submission.php

